\title{
Mauro Zonta (1968-2017)
}

Capita raramente di poter conoscere una persona che con il suo entusiasmo riesca a suscitare altrettanto entusiasmo verso lo studio e la scoperta, che con estrema umiltà e nobile gentilezza, del tutto gratuite, riesca a coinvolgere studenti e dottorandi, ricercatori e colleghi, in quella basilare e umana impresa che è il domandare, il ricercare, lo scoprire. Per tutte le persone che hanno avuto la grande fortuna di conoscerlo personalmente, Mauro Zonta è stato lui stesso una scoperta, una persona incredibilmente bella, uno studioso di altissimo livello e, per tanti, un caro amico. Coloro che non hanno avuto questa fortuna, hanno potuto leggere $\mathrm{i}$ suoi tantissimi contributi di ineccepibile precisione e vastissimo interesse, che hanno riplasmato e, sotto molteplici aspetti, riscritto la storia stessa del pensiero ebraico e latino.

Incessabile fonte di ispirazione, Mauro era una persona eccezionale nel senso più proprio di questo termine. Spesso riservato, fino all'ultimo non si è mai negato a partecipare alla più piccola iniziativa o contributo che gli fossero chiesti. Entusiasta del suo lavoro e delle sue ricerche, ad ogni incontro Mauro riusciva a suscitare il più grande interesse del pubblico, che lo ascoltava con vivida attenzione, trasportato in un altro tempo, fatto di testi e dispute, incontri e scontri tra dottrine e culture. Ma era a margine dei convegni, nelle chiacchierate scherzose e nei discorsi più vari, che Mauro mostrava il suo volto più autentico, il suo palpabile e profondo interesse per quella miriade di sviluppi opposti che costituisce la storia del pensiero umano, e quell'altrettanto intricata congerie di opinioni e storie che è la storia personale di ciascuno di noi. E così, da un incontro anche casuale o terzo, nuove idee e progetti venivano partoriti, in un'incessante tensione verso la scoperta e la comprensione.

La tragica notizia della prematura morte di Mauro ci lascia sconvolti e feriti. Non ci sono parole per esprimere il cordoglio, per spiegare a sufficienza la terribile perdita umana e scientifica che tutto ciò ci impone. In questo momento ci stringiamo alla famiglia di Mauro, uniti al loro dolore: è l'intera comunità accademica a farlo. Gli innumerevoli risultati raggiunti dai suoi studi sono e saranno dei preziosissimi semi da coltivare con cura. I suoi allievi e i suoi amici manterranno radicato il ricordo e l'eredità scientifica e umana di una persona così eccezionale come Mauro, che ha toccato in vario modo l'animo più profondo di tutti noi. $\mathrm{E}$ sebbene siamo coscienti che questa grande e vasta eredità perdurerà per lungo tempo, e che $\mathrm{i}$ contributi di Mauro continueranno a indirizzare gli studi di tutti noi, questa dolce consapevolezza tuttavia non ci strappa al dolore di una scomparsa così prematura, e al vuoto che ne consegue.

LOS EDITORES 